\title{
Synthesis of Nonlinear Optical Side-Chain Polymers Containing the Carbazolyldinitrophenylhydrazone Moiety by Polymer Reaction
}

\author{
Dong Wook KIM, ${ }^{\dagger}$ Soo Young PARK, and Sung Il Hong \\ Department of Fiber and Polymer Science, Seoul National University, \\ San 56-1, Shinlim-dong, Kwanak-ku, Seoul 151-742, Korea
}

(Received June 27, 1998)

\begin{abstract}
Side-chain polymer containing the carbazolyldinitrophenylhydrazone moiety was synthesized for nonlinear optical (NLO) applications. Because free radical polymerization resulted in molecular weight limitation due to the chain transfer nature of the NLO active chromophore, to prepare a high molecular weight polymer, a polymer reaction was chosen to functionalize a preformed aldehyde prepolymer. This prepolymer (P1) was synthesized by the reaction of 6-(3-formylcarbazol-9-yl)-hexyl methacrylate (M1) with methyl methacrylate (MMA). Successful polymerization was confirmed by ${ }^{1} \mathrm{H}$ and ${ }^{13} \mathrm{C}$ NMR spectroscopy and the molecular weight of the polymer was determined by gel permeation chromatography analysis. Polymer reaction of P1 with 2,4-dinitrophenyl hydrazine was carried out to prepare the NLO polymer (P2) and the reaction was monitored by NMR spectroscopy. This NLO polymer was soluble in common solvent and high optical quality thin film could be easily prepared for NLO activity measurement. The electro-optic coefficient $\left(r_{33}\right)$ of the polymer was determined $1.4 \mathrm{pm} \mathrm{V}^{-1}$ at poling field of $80 \mathrm{~V} \mu \mathrm{m}^{-1}$.
\end{abstract}

KEY WORDS Carbazolyldinitrophenylhydrazone / Nonlinear Optical Polymer / Polymer Reaction /

Nonlinear optical (NLO) effect means that the optical fields of high intensity produce the polarization in proportion to the product of two or more of the applied fields. This effect is expected to be used in the field of optical communication, optical switching, and optical signal processing..$^{1-4}$ Among various NLO materials, the poled side-chain NLO polymer is currently considered the most promising due to high chromophore content, improved temporal stability, good processibility, and easy structural modification. ${ }^{5}$

The main issues of side-chain NLO polymers are generally that the NLO chromophores should be designed to have effective $\pi$-conjugation capped at one end with electron donor and at the other end with electron acceptor to impart a high molecular hyperpolarizability $(\beta) .^{6,7}$ And NLO polymers should possess high glass transition temperature $\left(T_{\mathrm{g}}\right)$ to prevent the dipolar relaxation of poled system.

This work tried to enhance NLO activity and temporal stability by introducing carbazole group and dinitrophenylhydrazone moiety into the chromophore structure. Thermally stable carbazole group was used as an electron donor group and dinitrophenylhydrazone unit was incorporated into the chromophore structure as an electron acceptor. Particularly, the hydrazone chromophores are known to have a significantly larger static first order hyperpolarizability $(\beta)$ than the corresponding diene derivatives. ${ }^{2}$

To prepare target NLO polymer (P2), at first trial, we followed route I (Scheme 1). However, there is a difficulty in the synthesis of high molecular weight polymer by free radical polymerization. The chain transfer nature of this NLO active monomer (M2) presumably limits the molecular weight of the final polymer. ${ }^{8,9}$ Therefore, to prepare a high molecular weight polymer, a polymer reaction was chosen to functionalize a preformed aldehyde polymer (P1), which could be polymerized to high molecular weight (route II). Successful polymerization was confirmed by ${ }^{1} \mathrm{H}$ and ${ }^{13} \mathrm{C}$ NMR spectroscopy and the molecular weight of the polymer was determined by gel permeation chromatography analysis. NLO activity of the polymer was estimated by measuring the

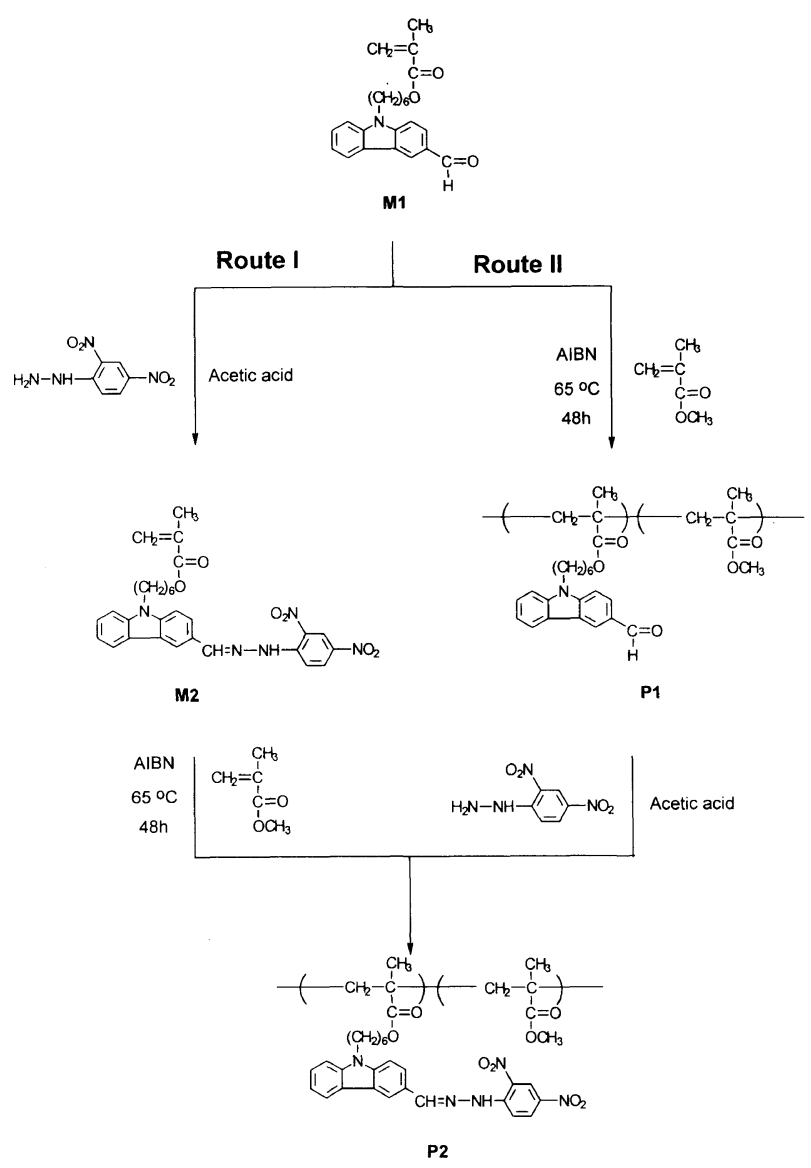

Scheme 1. Synthesis of the side-chain NLO polymer.

\footnotetext{
${ }^{\dagger}$ Present address: Hybrid Polymer Research Center, Korea Institute of Science and Technology, P.O. Box 131, Cheongryang, Seoul 130-650, Korea.
} 

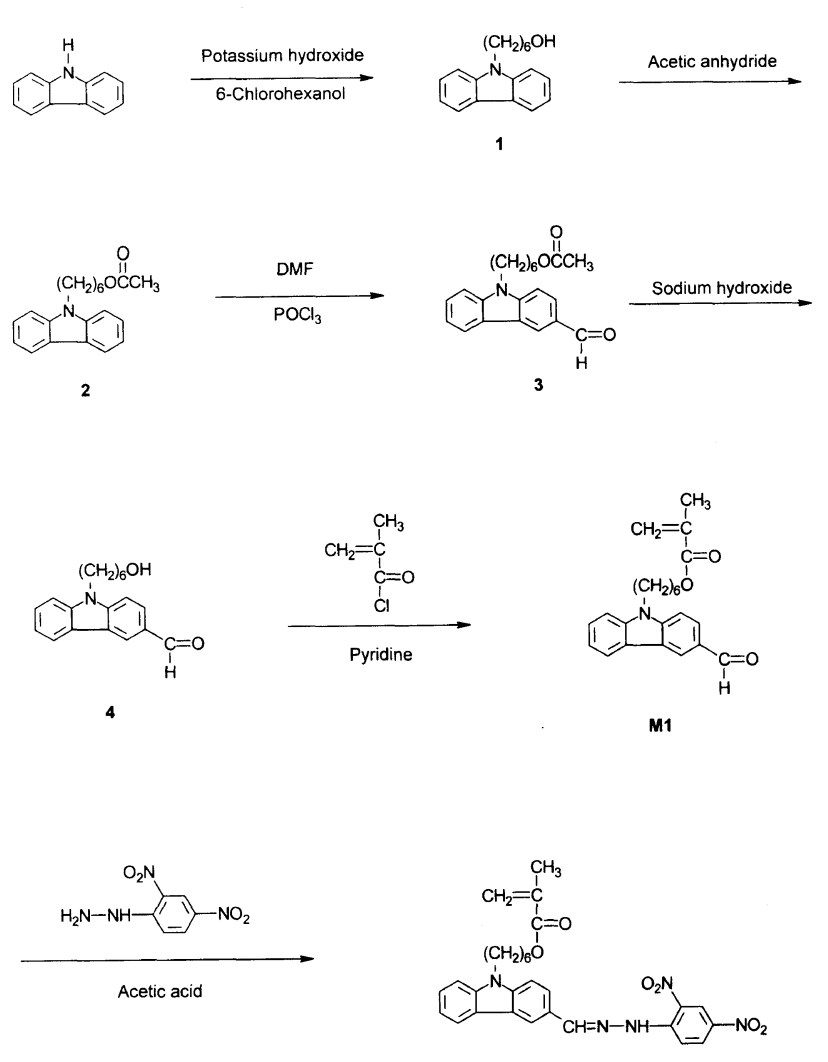

M2

Scheme 2. Synthesis of the NLO monomer.

electro-optic coefficient.

\section{EXPERIMENTAL}

\section{Materials}

Organic solvents were purified by distillation over the suitable dehydrating reagents just before use. $9 \mathrm{H}$ Carbazole purchased from Aldrich Chem. Co. was purified by recrystallization from ethanol. Commercially available 6-chlorohexanol and 2,4-dinitrophenylhydrazine were used as received. Methacryloyl chloride purchased from Aldrich Chem. Co. was purified by distillation at reduced pressure.

\section{Synthesis of Monomer}

The synthetic route of the NLO monomer is illustrated in Scheme 2. 9-(6-Hydroxyhexyl)-9H-carbazol-3-carbaldehyde (4) was key material in the synthesis of the monomer and it was prepared by the literature method. ${ }^{10}$

\section{6-(3-Formyl-carbazol-9-yl)-hexyl Methacrylate (M1)}

$5.91 \mathrm{~g}(0.02 \mathrm{~mol})$ of compound 4 were dissolved in freshly distilled tetrahydrofuran (THF) $(40 \mathrm{ml})$. Pyridine $(1.61 \mathrm{ml})$ and a trace amount of 2,6-di-t-butyl-4-methyl phenol (polymerization inhibitor) were added to the solution. The solution was cooled in ice bath and 1.95 $\mathrm{ml}(0.02 \mathrm{~mol})$ of methacryloyl chloride were added slowly under nitrogen atmosphere. After stirring at $0^{\circ} \mathrm{C}$ for $30 \mathrm{~min}$, the solution was heated at $40^{\circ} \mathrm{C}$ for $24 \mathrm{~h}$. The resulting solution was poured into sodium bicarbonate solution and washed with water. The mixture was extracted with ethyl acetate and dried over $\mathrm{MgSO}_{4}$. The solvent was removed at reduced pressure and the residue was purified by silica gel column chromatography ( $n$ hexane/ethyl acetate $=3 / 1$ ) to give viscous oil (yield $82 \%)$. IR ( $\mathrm{KBr}$ window, $\left.\mathrm{cm}^{-1}\right) ; 2816$ and $2735\left(v_{\mathrm{C}-\mathrm{H}}\right.$ of aldehyde), $1714\left(v_{\mathrm{C}=\mathrm{O}}\right.$ of ester $), 1686\left(v_{\mathrm{C}=\mathrm{O}}\right.$ of aldehyde). ${ }^{1} \mathrm{H}$ NMR $\left(\mathrm{CDCl}_{3}\right) ; \delta(\mathrm{ppm})=9.9(\mathrm{~s}, 1 \mathrm{H}$, aldehyde proton), $8.4(\mathrm{~s}, 1 \mathrm{H}), 7.9(\mathrm{~d}, 1 \mathrm{H}), 7.6(\mathrm{~d}, 1 \mathrm{H})$, $7.3-7.1(\mathrm{~m}, 4 \mathrm{H}), 5.8$ and $5.3(2 \mathrm{H}$, vinyl protons), 4.1 $\left(\mathrm{t}, 2 \mathrm{H}, \mathrm{OCH}_{2}\right), 3.9\left(\mathrm{t}, 2 \mathrm{H}, \mathrm{NCH}_{2}\right), 1.9(\mathrm{~s}, 3 \mathrm{H}$, $\left.\mathrm{CH}_{3}\right), 1.7-1.2\left(\mathrm{~m}, 8 \mathrm{H},\left(\mathrm{CH}_{2}\right)_{4}\right) .{ }^{13} \mathrm{C} \mathrm{NMR}\left(\mathrm{CDCl}_{3}\right) ; \delta$ $(\mathrm{ppm})=192.0(1 \mathrm{C}$, aldehyde carbon $), 167.7(1 \mathrm{C}$, carbonyl carbon), 136.8 and 125.7 (2C, vinyl carbons) 144.3, 141.4, $128.8,127.4,127.1,124.2,123.3,123.3,121.1,120.7$, 109.7, 109.2 (12C, carbazole ring carbons), 64.8, 43.5, 29.2, 28.8, 27.2, 26.2 (6C, aliphatic carbons), 18.7 (1C, $\mathrm{CH}_{3}$ ).

\section{6-[3-(2,4-Dinitrophenyl-hydrazonomethyl)-carbazol- 9-yl]-hexyl Methacrylate (M2)}

To a stirred solution of M1 (3.63 g, $0.01 \mathrm{~mol})$ in THF were added 2,4-dinitrophenyl hydrazine $(1.98 \mathrm{~g}, 0.01 \mathrm{~mol})$ and a trace amount of 2,6-di-t-butyl-4-methyl phenol. When the mixture was dissolved, a small amount of glacial acetic acid was slowly added, and the solution was stirred at $40^{\circ} \mathrm{C}$ for $24 \mathrm{~h}$. The resulting solution was cooled to room temperature and precipitated into ethanol. The precipitate was collected by filtration, and washed with ethanol several times. The product was dried under vacuum to give red solid (yield $64 \%$ ). mp $210^{\circ} \mathrm{C}$. IR (KBr pellet, $\left.\mathrm{cm}^{-1}\right) ; 3287\left(v_{\mathrm{N}-\mathrm{H}}\right), 1716\left(v_{\mathrm{C}=\mathrm{O}}\right.$ of ester group), 1516 and 1334 ( $\left.v_{\text {nitro group }}\right) .{ }^{1} \mathrm{H} \mathrm{NMR}\left(\mathrm{CDCl}_{3}\right) ; \delta$ $(\mathrm{ppm})=11.3(\mathrm{~s}, 1 \mathrm{H}, \mathrm{N}-\mathrm{H}), 9.1(\mathrm{~d}, 1 \mathrm{H}), 8.4(\mathrm{~s}, 1 \mathrm{H}), 8.3$ $(\mathrm{d}, 1 \mathrm{H}), 8.2(\mathrm{~s}, 1 \mathrm{H}), 8.1(\mathrm{~d}, 1 \mathrm{H}), 7.8(\mathrm{~d}, 1 \mathrm{H}), 7.5(\mathrm{t}, 1 \mathrm{H})$, $7.4(\mathrm{dd}, 2 \mathrm{H}), 7.2(\mathrm{t}, 1 \mathrm{H}), 6.0$ and $5.5(2 \mathrm{H}$, vinyl protons), $4.3\left(\mathrm{t}, 2 \mathrm{H}, \mathrm{OCH}_{2}\right), 4.0\left(\mathrm{t}, 2 \mathrm{H}, \mathrm{NCH}_{2}\right), 1.8\left(\mathrm{~s}, 3 \mathrm{H}, \mathrm{CH}_{3}\right)$, $1.7-1.3\left(\mathrm{~m}, 8 \mathrm{H},\left(\mathrm{CH}_{2}\right)_{4}\right)$. Anal. Calcd for $\mathrm{C}_{29} \mathrm{H}_{29} \mathrm{~N}_{5} \mathrm{O}_{6}$ : C, 64.07; H, 5.38; N, 12.89. Found: C, 63.85; H, 5.05; N, 12.87 .

\section{Synthesis of Polymers}

Preparation of polymers was carried out by routes I and II illustrated in Scheme 1.

Preparation of NLO Polymer (P2a-I, P2b-I) by Route I

To prepare polymers P2a-I and P2b-I, monomer M2 and methyl methacrylate (MMA) were dissolved in $N$ methylpyrrollidone (NMP) with $\alpha, \alpha^{\prime}$-azobis(isobutyronitrile) (AIBN) as an initiator. The feed ratio of $\mathbf{M} 2$ to MMA was $1: 3$ in P2a-I and 1:9 in P2b-I. AIBN was used as much as $5 \mathrm{~mol} \%$ to the total monomers. The resulting red clear solution was thoroughly degassed by several freeze-pump-thaw cycles and heated in a sealed ampoule at $65^{\circ} \mathrm{C}$ for 2 days. The solution was cooled and poured into vigorously agitated ethanol to precipitate a solid. To purify the product, several reprecipitations from NMP to ethanol were performed.

\section{Preparation of Aldehyde Prepolymer $(\boldsymbol{P 1} \boldsymbol{a}, \boldsymbol{P} \mathbf{1 b})$}

The preparation of prepolymer is described in Table $\mathrm{I}$ and typical procedure is as follows. $0.70 \mathrm{~g}(1.9 \mathrm{mmol})$ of monomer M1, $1.74 \mathrm{~g}$ of MMA, and $31.1 \mathrm{mg}(1 \mathrm{~mol} \%$ to the total monomers) of AIBN as an initiator were dissolved in THF. The solution was thoroughtly de- 
Table I. Preparation of and polymerization results for aldehyde prepolymers

\begin{tabular}{|c|c|c|c|c|c|}
\hline \multirow{2}{*}{ Polymer } & Feed ratio & Conversion & Composition $^{\mathrm{a}}$ & \multirow{2}{*}{$M_{w}^{\mathrm{b}}$} & \multirow{2}{*}{$M_{w} / M_{n}^{\mathrm{b}}$} \\
\hline & M1/MMA & $\%$ & M1/MMA & & \\
\hline P1a & $1 / 3$ & 90 & $1 / 2.98$ & 39300 & 2.10 \\
\hline P1b & $1 / 9$ & 76 & $1 / 9.77$ & 54700 & 1.81 \\
\hline
\end{tabular}

${ }^{a}$ Determined by ${ }^{1} \mathrm{H}$ NMR spectroscopy. ${ }^{\mathrm{b}}$ Determined by GPC with polystyrene standard in THF eluent.

gassed by several freeze-pump-thaw cycles and heated in a sealed ampoule at $65^{\circ} \mathrm{C}$ for 2 days. The solution was cooled and poured into vigorously agitated ethanol to precipitate solid. The product was purified by repeated reprecipitations from THF into ethanol. After filtration and vacuum drying, a white colored P1b $(1.86 \mathrm{~g})$ was obtained.

\section{Preparation of NLO Polymer (P2a-II, P2b-II) by Polymer} Reaction on Aldehyde Prepolymer

To prepare NLO polymer by route II, 2,4-dinitrophenylhydrazine was added to a stirred solution of aldehyde prepolymer in THF. P1a was the prepolymer for P2a-II and P1b for P2b-II. 2,4-Dinitrophenylhydrazine was used 5 times the amount of the aldehyde group in the prepolymer. When the resulting mixture dissolved, a small amount of glacial acetic acid was slowly added and then heated. After the reaction was completed, the solution was cooled and precipitated into ethanol. The precipitate was collected by filtration, purified by repeated reprecipitations from $\mathrm{THF}$ to ethanol. The product was dried under vacuum to yield red solid.

\section{Instrumentation}

FT-IR spectra were recorded on a Midac FT-IR spectrophotometer. ${ }^{1} \mathrm{H}$ NMR and ${ }^{13} \mathrm{C}$ NMR spectra were obtained with a Bruker AMX 300 spectrometer. Elemental analysis was carried out with a Carlo Erba 116 Elemental Analyzer with a LECO CHNS-932. UVVis spectra were obtained with a Shimadzu UV-2101PC double beam spectrophotometer. Gel permeation chromatography (GPC) analysis of polymers was performed at a flow rate $1.0 \mathrm{ml} \mathrm{min}^{-1}$ in $\mathrm{THF}$ at $30^{\circ} \mathrm{C}$ using Waters HPLC component system equipped with five Ultra- $\mu$-styragel ${ }^{\circledR}$ columns $\left(2 \times 10^{5}, 10^{4}, 10^{3}, 500 \AA\right)$. DSC measurements were performed on a Perkin Elmer DSC7.

\section{Fabrication of Polymer Films}

NLO polymer was dissolved in tetrachloroethane $(10 \mathrm{wt} \%)$ and the solution was filtered through a Teflon membrane filter $(0.2 \mu \mathrm{m})$. Thin polymer films were spin-coated onto slide glass and indium-tin oxide (ITO) coated glass. Residual solvent was removed by heating the films in vacuum oven for several days. Film thickness was measured with a TENCOR P-10 profilometer.

\section{Measurement of Electro-Optic Coefficient}

For electro-optic coefficient measurement, contact poling of polymer film was carried out. A gold electrode was sputter-coated in $10^{-6}$ torr vacuum over the polymer film backed with ITO glass. To align the chromo- (a)

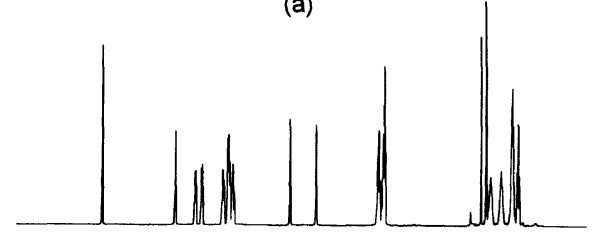

(b)

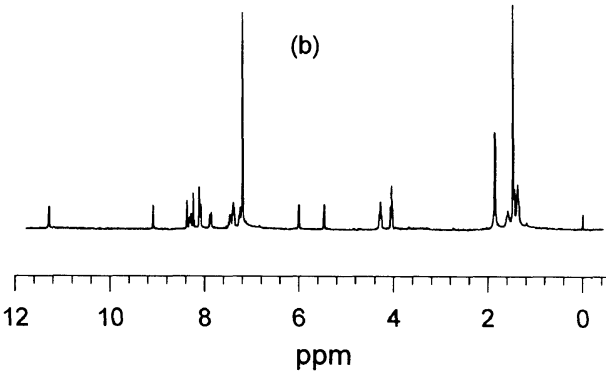

Figure 1. ${ }^{1} \mathrm{H}$ NMR spectra of monomers, (a) M1 and (b) M2.

phores, the sample was heated to $T_{\mathrm{g}}$ and a poling field was applied to the gold electrode. After $15 \mathrm{~min}$, the film was cooled to room temperature and the poling field was turned off. The electro-optic coefficient $\left(r_{33}\right)$ of poled polymer was measured at $632.8 \mathrm{~nm}$ (He-Ne laser) using a simple reflection method proposed by Teng et al. ${ }^{11} \mathrm{~A}$ Soleil-Babinet compensator was used to bias the dc intensity at the half-maximum intensity. The amplitude of modulated intensity was detected using a lock-in amplifier, which was used to calculate $r_{33}$.

\section{RESULTS AND DISCUSSION}

\section{Synthesis of Monomers}

The key material in the synthesis of the monomer was compound 4. This compound was found to be synthesized specifically by Vilsmeier formylation of compound 2 followed by deprotection with base. Compound 4 was fully identified by IR and NMR spectroscopy in the previous report. ${ }^{10}$

M1 was prepared through the reaction of 4 with methacryloyl chloride. The structure of M1 was confirmed by IR and NMR spectroscopy. In IR spectrum, the $\mathrm{C}=\mathrm{O}$ stretching bands of ester group and aldehyde group were observed at $1739 \mathrm{~cm}^{-1}$ and $1684 \mathrm{~cm}^{-1}$, respectively. ${ }^{1} \mathrm{H}$ NMR spectrum as shown in Figure 1(a) apparently shows the aldehyde proton at $9.9 \mathrm{ppm}$ and protons attached to vinyl group at 5.8 and $5.3 \mathrm{ppm}$, indicating the successful introduction of vinyl group together with an intact aldehyde group. The normal and DEPT-135 spectra in Figure 2 confirmed the structure of M1 more accurately. The single peak at $192.0 \mathrm{ppm}$ must arise from aldehyde carbon (C13). Comparing Figures 2(a) and (b), it is noted that quarternary carbons, C1, C6, C7, C9, C12, C20, and C21 disappeared in DEPT-135 spectrum. The methylene carbon in vinyl group, C22, was recorded as a negative peak at $126.9 \mathrm{ppm}$ in DEPT-135 spectrum. Aliphatic carbons $(\mathrm{C} 14-\mathrm{C} 19)$ in spacer also give rise to negative peaks at $64-26 \mathrm{ppm}$ because they are methylene carbons.

M2 was obtained by reaction of M1 with 2,4-dinitrophenylhydrazine. This reaction should be carefully carried out to protect the susceptible vinyl group. The 

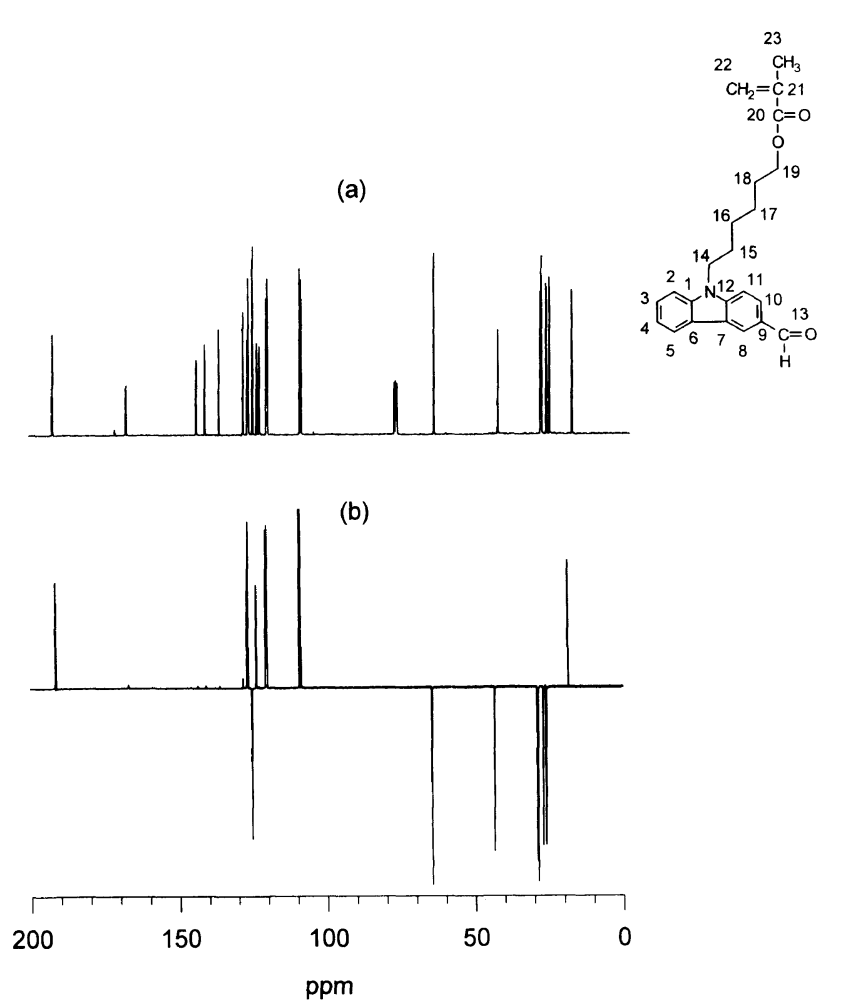

Figure 2. ${ }^{13} \mathrm{C}$ NMR spectra of monomer M1, (a) normal and (b) DEPT-135.

structure of $\mathbf{M} 2$ was confirmed by IR and ${ }^{1} \mathrm{H}$ NMR spectroscopy. In IR spectrum, $\mathrm{N}-\mathrm{H}$ stretching band at $3287 \mathrm{~cm}^{-1}$ and nitro group stretching band at 1516 and $1334 \mathrm{~cm}^{-1}$ were observed, together with the disappearance of characteristic stretching vibration of aldehyde group. Figure 1(b) shows ${ }^{1} \mathrm{H}$ NMR spectrum of M2. The distinct sharp peaks due to the proton in amine group at $11.3 \mathrm{ppm}$ and protons attached to the vinyl group at $6.0 \mathrm{ppm}$ and $5.5 \mathrm{ppm}$ revealed the successful synthesis of monomer M2.

\section{Synthesis of the NLO Polymer by Route I}

To prepare polymers P2a-I and P2b-I, the reaction of $\mathrm{M} 2$ and MMA using AIBN as an initiator in NMP solvent was performed. Due to chain transfer of the vinyl monomer M2 with two nitro group, however, conversion and molecular weight of polymers were very low by free radical polymerization method. By ${ }^{1} \mathrm{H}$ NMR spectroscopy, in case of preparation of P2a-I (M2/MMA =1/3), the spectrum of the polymerization product had little difference from that of the corresponding monomer. The higher the ratio of MMA, the greater was the degree of polymerization, but the peaks of vinyl group did not disappear indicating presence of the unreacted monomers. We tried to isolate the polymer product, but the residue monomer was not thoroughly removed probably due to the low molecular weight of the product.

\section{Synthesis of Aldehyde Prepolymer}

Aldehyde prepolymers P1a and P1b were prepared by reaction of M1 with MMA. The polymerization results of the aldehyde prepolymers are summarized in Table I. Aldehyde prepolymers could be prepared in very good yields $(80-90 \%)$ and weight-average molecular weight was found to be high $(40000-55000)$, probably because

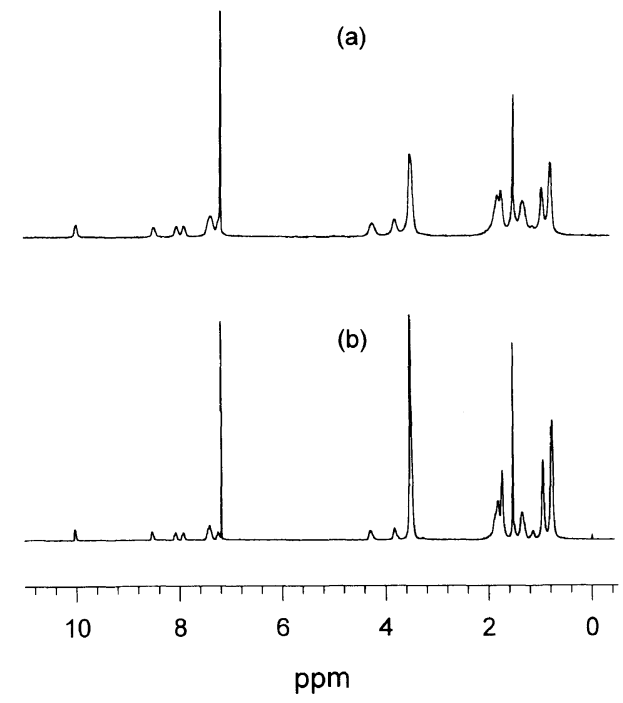

Figure 3. ${ }^{1} \mathrm{H}$ NMR spectra of aldehyde prepolymers, (a) P1a and (b) P1b.

monomer M1 had little chain transfer. Particularly, in ${ }^{1} \mathrm{H}$ NMR spectrum as shown Figure 3, the copolymer composition could be determined by comparing $-\mathrm{NCH}_{2}$ and $-\mathrm{OCH}_{2}$ peaks originating from $\mathrm{M1}$ at $3.9-4.1 \mathrm{ppm}$ with $-\mathrm{OCH}_{3}$ peak from MMA at $3.2 \mathrm{ppm}$. The copolymer composition of P1a was similar to the monomer ratio in the feed, but in P1b the content of MMA was a little higher than the feed.

The structure of the polymer was confirmed by ${ }^{1} \mathrm{H}$ and ${ }^{13} \mathrm{C}$ NMR spectroscopy. Figure 3 shows ${ }^{1} \mathrm{H}$ NMR spectra of P1a and P1b. From the spectra, it is noted that the peaks of vinyl protons at 6.1 and $5.5 \mathrm{ppm}$ of the monomer M1 disappeared, together with rather broad signals typical of polymeric materials which means successful polymerization. In Figure 4, Dept-135 spectra of M1 and P1a are shown. The negative peak at 125.7 ppm in M1 spectrum must be from methylene carbon of vinyl group, while in P1a spectrum the peak of the corresponding methylene carbon shifted toward upfield $(54.8 \mathrm{ppm})$ indicating successful polymerization. Also, the carbon in $-\mathrm{OCH}_{3}$ from MMA was observed at $52.2 \mathrm{ppm}$ in Figure 4(b). In particular, peak at $10.0 \mathrm{ppm}$ in ${ }^{1} \mathrm{H}$ NMR spectrum and the peak at $192.1 \mathrm{ppm}$ in DEPT-135 spectrum of P1a must be attributed to the aldehyde proton and carbon, confirming the intact aldehyde group after polymerization.

\section{Synthesis of NLO Polymer by Polymer Reaction on the Aldehyde Prepolymer}

In polymer reaction, solvation of polymer is a key issue and use of solvent, which can maximize the polymer solution hydrodynamic volume, allows chain extension of the backbone and hence minimize steric hindrance. THF used in this work is a good polar solvent for our polymers, allowing the polymer reaction preferred. To obtain fully functionalized NLO polymer, 2,4-dinitrophenylhydrazine was used 5 times the amount of the aldehyde group in prepolymer. In preparation P2a-II, however, as the reaction progressed the product began to behave as a gel, which proved to be unprocessable. But, the preparation of $\mathbf{P 2} \mathbf{b}-\mathbf{I I}$ by polymer reaction on the prepolymer P1b was performed successfully and this 
(a)
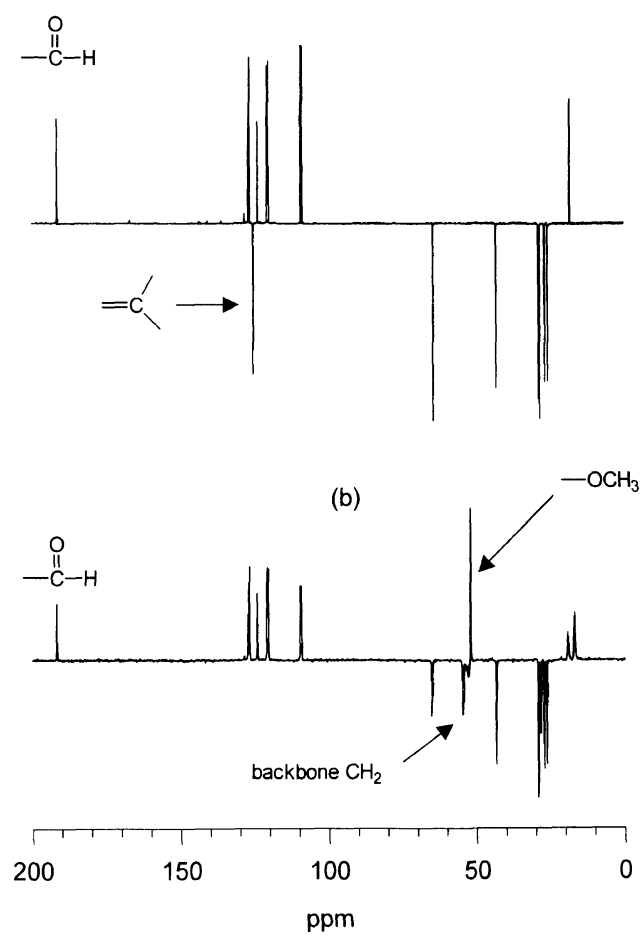

Figure 4. ${ }^{13} \mathrm{C}$ NMR DEPT-135 spectra of (a) M1 and (b) P1a.

(a)
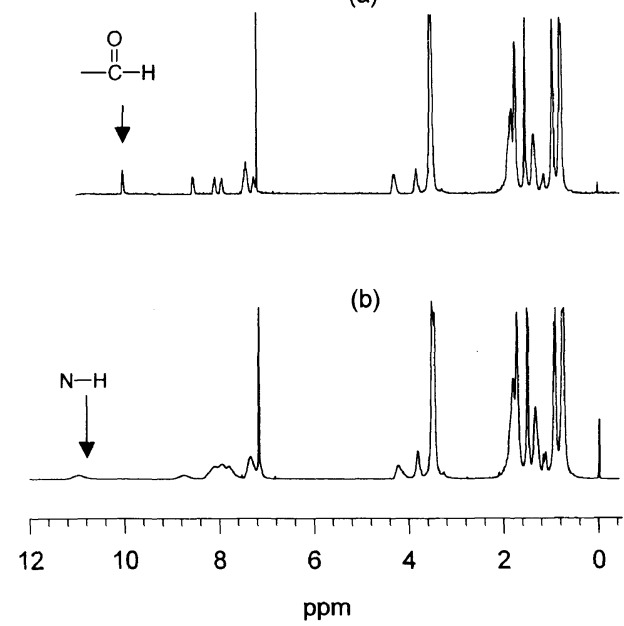

Figure 5. ${ }^{1} \mathrm{H}$ NMR spectra of (a) prepolymer P1b and (b) NLO polymer P2b-II.

was confirmed by ${ }^{1} \mathrm{H}$ NMR spectroscopy. In Figure 5, the aldehyde proton peak in $\mathbf{P 1 b}$ at $10 \mathrm{ppm}$ has disappeared along with the advent of $\mathrm{N}-\mathrm{H}$ peak in P2b-II at $11 \mathrm{ppm}$. This strongly confirms the full conversion of aldehyde group to dinitrophenylhydrazone unit. From the ${ }^{1} \mathrm{H}$ NMR spectroscopy, the content of incorporated dinitrophenylhydrazone chromophore in P2b-II was found to be about $9.3 \%$.

\section{Thermal Property and NLO Activity of Polymer}

DSC thermograms of the polymers in Figure 6 show glass transition temperature $\left(T_{\mathrm{g}}\right)$ of $\mathbf{P 1 b}$ and P2b-II. It should be noted that incorporation of hydrazone chromophore result in sharp increase of $T_{\mathrm{g}}$. This $T_{\mathrm{g}}$ of $\mathbf{P 2 b} \mathbf{b}$ II is quite high considering the low content of NLO

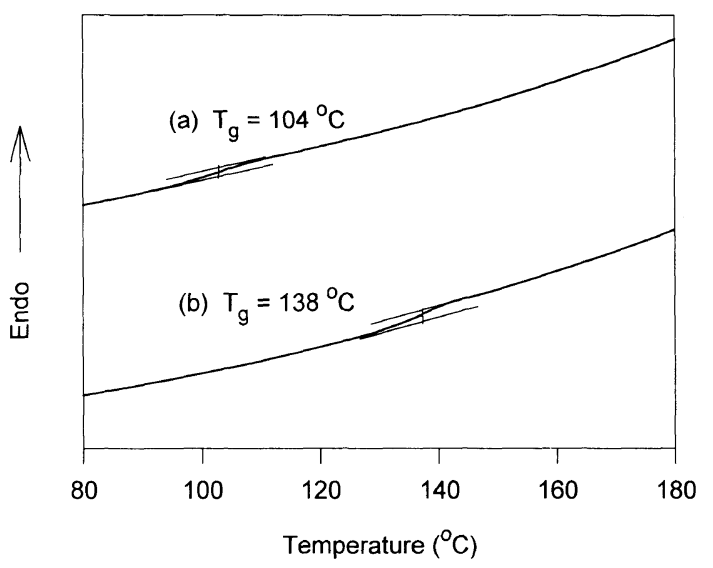

Figure 6. DSC thermograms of (a) P1b and (b) P2b-II.

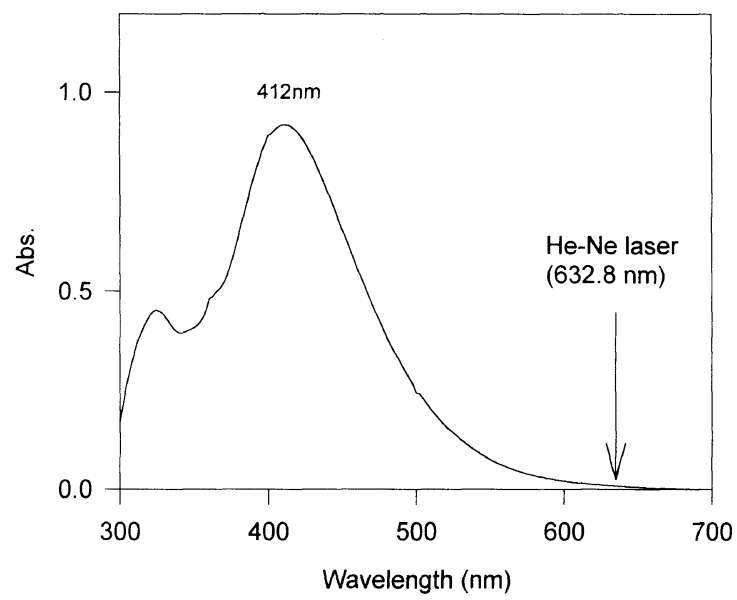

Figure 7. Absorption spectrum of polymer P2b-II.

chromophore (9.3\%) and long spacer (6 methylene units). This shows the carbazolyldinitrophenylhydrazone chromophore can give high thermal stability to the polymer.

Polymer P2b-II was very soluble in common solvent like THF, chloroform, dichloroethane, and tetrachloroethane. High quality thin film $(1.5 \mu \mathrm{m})$ of the sidechain NLO polymer P2b-II could be easily prepared by spin-coating of the polymer solution in tetrachloroethane onto an ITO glass substrate. Dipole alignment and the second-order nonlinearity of the polymer could be induced by contact poling. The sample was poled at $150^{\circ} \mathrm{C}$ with an applied dc electric field of $80 \mathrm{~V} \mu \mathrm{m}^{-1}$. $r_{33}$ of the polymer P2b-II was $1.4 \mathrm{pm} \mathrm{V}^{-1}$. This electro-optic coefficient is virtually non-resonant value because the polymer does not absorb at the He-Ne laser wavelength $(632.8 \mathrm{~nm})$ as shown in Figure 7 . The value was a little low presumably due to the low content of NLO chromophore. From the further structural optimization, it is expected that NLO activity of our polymer will be promoted. Because this polymer possesses photoconductive moiety in the structure, possible photorefractive effect can be revealed. ${ }^{12}$

\section{CONCLUSION}

The carbazolyldinitrophenylhydrazone moiety was used as NLO chromophore to enhance NLO activity and thermal stability. To prepare side-chain NLO polymer, 
the reaction of NLO monomer and MMA was tried, but conversion and molecular weight of polymer were very low due to chain transfer of the NLO active chromophore. Therefore, to prepare a high molecular weight NLO polymer, a polymer reaction was chosen to functionalize a preformed aldehyde polymer. Aldehyde prepolymers were synthesized in very good yields and weight-average molecular weights were $40000-55000$. The target NLO polymer was successfully prepared by reaction of prepolymer with 2,4-dinitrophenylhydrazine. The content of hydrazone chromophore incorporated could be evaluated by ${ }^{1} \mathrm{H}$ NMR spectroscopy. Optical quality thin film of the side-chain NLO polymer P2b-II could be easily prepared by spin-coating the polymer solution in tetrachloroethane onto an ITO glass. The film was poled at $150^{\circ} \mathrm{C}$ with an applied dc electric field of $80 \mathrm{~V} \mu \mathrm{m}^{-1}$ and the electro-optic coefficient was found to be $1.4 \mathrm{pm} \mathrm{V}^{-1}$. Further structural optimization to enhance NLO activity is in progress.

Acknowledgments. We thank Dr. J. K. Kim in Korea
Institute of Science and Technology for helpful discussion. We would also like to thank Dr. N. Kim and Mr. H. S. Moon for the measurement of NLO property.

\section{REFERENCES}

1. W. Nie, Adv. Mater., 5, 520 (1993).

2. J. Zyss, "Molecular Nonlinear Optics," Academic Press, New York, N.Y., 1994.

3. G. Boyd, J. Opt. Soc. Am. B, 6, 685 (1989).

4. G. Möhlmann, Synth. Met., 37, 207 (1990).

5. D. M. Burland, R. D. Miller, and C. A. Walsh, Chem. Rev., 94, 31 (1994)

6. S. R. Marder and J. W. Perry, Adv. Mater., 5, 804 (1993).

7. A. Buckly, Adv. Mater., 4, 153 (1992).

8. K. Ivan, D. Danica, S. Jaroslav, and N. Stanislav, Polym. Bull., 35, 315 (1995).

9. M. C. Iain, D. M. Ronald, K. Richard, L. Thomas, and M. Hongtai, Macromol. Chem. Phys., 197, 687 (1996).

10. D. W. Kim, S. I. Hong, S. Y. Park, and N. Kim, Bull. Korean Chem. Soc., 18, 198 (1997).

11. C. C. Teng and H. T. Man, Appl. Phys. Lett., 56, 1754 (1990).

12. D. W. Kim, H. Moon, S. Y. Park, and S. I. Hong, Reactive \& Functional Polymers, in press. 